\title{
On the Korobov and Changhee mixed-type polynomials and numbers
}

\author{
Byung Moon Kim ${ }^{\mathrm{a}}$, Jeong Gon Lee ${ }^{\mathrm{b}}$, Lee-Chae Jang ${ }^{\mathrm{c}, *}$, Sangki Choi ${ }^{\mathrm{d}}$ \\ ${ }^{a}$ Department of Mechanical System Engineering, Dongguk University, Gyeongju, 780-714, Korea. \\ ${ }^{b}$ Division of Mathematics and Informational Statistics and Nanoscale Science and Technology Institute, Wonkwang University, Iksan \\ 570-749, Republic of Korea. \\ ${ }^{c}$ Graduate School of Education, Konkuk University, Seoul 143-701, Republic of Korea. \\ ${ }^{d}$ Department of Mathematics Education, Konkuk University, Seoul 143-701, Korea.
}

\begin{abstract}
By using the Bosonic p-adic integral, Kim et al. [D. S. Kim, T. Kim, H.-I. Kwon, J.-J. Seo, Adv. Stud. Theor. Phys., 8 (2014), 745-754] studied some identities of the Korobov and Daehee mixed-type polynomials. In this paper, by using the fermionic p-adic integral, we define the Korobov and Changhee mixed-type polynomials and give some interesting identities of those polynomials. (C)2017 All rights reserved.
\end{abstract}

Keywords: Korobov polynomials, Changhee polynomials, Korobov and Changhee mixed-type polynomials. 2010 MSC: 11B68, 11S40.

\section{Introduction}

Let $p$ be an odd prime number. Throughout this paper, $\mathbb{Z}_{p}, \mathbb{Q}_{p}$, and $\mathbb{C}_{p}$ denote the ring of $p$-adic integers, the filed of $p$-adic rational numbers, and the completion of algebraic closure of $\mathbb{Q}_{p}$, respectively. $\mathbb{Z}_{\mathrm{p}}$.

The $p$-adic norm $|\cdot|_{p}$ is normalized as $|p|_{p}=\frac{1}{p}$. Let $C\left(\mathbb{Z}_{p}\right)$ be the space of continuous functions on

For $f \in C\left(\mathbb{Z}_{p}\right)$, the fermionic $p$-adic integrals on $\mathbb{Z}_{p}$ is defined by Kim to be

$$
I_{-1}(f)=\int_{\mathbb{Z}_{p}} f(x) d \mu_{-1}(x)=\lim _{N \rightarrow \infty} \sum_{x=0}^{p^{N}-1} f(x)(-1)^{x}, \quad \text { see [1-21]). }
$$

From (1.1), it is well-known that

$$
\mathrm{I}_{-1}\left(\mathrm{f}_{1}\right)+\mathrm{I}_{-1}(\mathrm{f})=2 \mathrm{f}(0),
$$

where $f_{1}(x)=f(x+1)$. By using (1.2), we get

\footnotetext{
*Corresponding author

Email addresses: kbm713@dongguk.ac.kr (Byung Moon Kim), jukolee@wku.ac.kr (Jeong Gon Lee), lcjang@konkuk.ac.kr (Lee-Chae Jang), schoi@konkuk. ac.kr (Sangki Choi)

doi:10.22436/jmcs.017.03.05
} 


$$
\int_{\mathbb{Z}_{p}}(1-t)^{q y+x} d \mu_{-1}(y)=\frac{2}{(1-t)^{q}+1}(1-t)^{x} .
$$

Recall that the q-Chaghee polynomials are defined by the generating function

$$
\frac{2}{(1+t)^{q}+1}(1+t)^{x}=\sum_{x=0}^{\infty} C h_{n, q}(x) \frac{t^{n}}{n !}, \quad(\text { see }[20]) .
$$

From (1.3) and (1.4), we have

$$
\frac{2}{(1-t)^{q}+1}(1-t)^{x}=\sum_{n=0}^{\infty} C h_{n, q}(x)(-1)^{n} \frac{t^{n}}{n !} .
$$

By replacing $t$ by $1-e^{t}$ in (1.5), we have

$$
\begin{aligned}
\text { LHS of (1.5) } & =\frac{2}{1+e^{q t}} e^{x t} \\
& =\frac{2}{1+e^{q t}} e^{\frac{x}{q} q t} \\
& =\sum_{n=0}^{\infty} E_{n}\left(\frac{x}{q}\right) q^{n} \frac{t^{n}}{n !}
\end{aligned}
$$

and

$$
\begin{aligned}
\text { RHS of (1.5) } & =\sum_{n=0}^{\infty} C h_{n, q}(x)(-1)^{n} \frac{\left(1-e^{t}\right)^{n}}{n !} \\
& =\sum_{n=0}^{\infty} C h_{n, q}(x) \frac{1}{n !} n ! \sum_{m=n}^{\infty} S_{2}(m, n) \frac{t^{m}}{m !} \\
& =\sum_{m=0}^{\infty}\left(\sum_{n=0}^{m} C h_{n, q}(x) S_{2}(m, n)\right) \frac{t^{m}}{m !} .
\end{aligned}
$$

From (1.6) and (1.7), we obtain the following theorem.

Theorem 1.1. For $m \in \mathbb{N} \cup\{0\}$, we have

$$
E_{m}\left(\frac{x}{q}\right) q^{m}=\sum_{n=0}^{m} C h_{n, q}(x) S_{2}(m, n) .
$$

We observe that

$$
\begin{aligned}
\int_{\mathbb{Z}_{\mathfrak{p}}}(1-t)^{q y+x} d \mu_{-1}(y) & =\sum_{m=0}^{\infty} \int_{\mathbb{Z}_{\mathfrak{p}}}\left(\begin{array}{c}
q y+x \\
m
\end{array}\right)(-t)^{m} d \mu_{-1}(y) \\
& =\sum_{m=0}^{\infty} \int_{\mathbb{Z}_{p}}(q y+x)_{m} d \mu_{-1}(y)(-1)^{m} \frac{t^{m}}{m !}
\end{aligned}
$$

From (1.3), (1.5), and (1.8), we obtain the following theorem.

Theorem 1.2. For $m \in \mathbb{N} \cup\{0\}$, we have

$$
\int_{\mathbb{Z}_{p}}(q y+x)_{m} d \mu_{-1}(y)=C h_{m, q}(x) .
$$


The Stirling numbers of the first kind are defined by

$$
(x)_{n}=\sum_{l=0}^{n} S_{1}(n, l) x^{l}, \quad(n \geqslant 0) .
$$

The Stirling numbers of the second kind are defined by

$$
x^{n}=\sum_{l=0}^{n} S_{2}(n, l)(x)_{l}, \quad(n \geqslant 0) .
$$

By using the Bosonic p-adic integral, Kim et al. ([11, 12, 14]) studied some identities of the Korobov and Daehee mixed-type polynomials. In this paper, we observe the Korobov and Daehee mixed-type polynomials in a slightly different way and use the Fermionic p-adic integral in stead of the Bosonic p-adic integral. From the Fermionic p-adic integral, we define the Korobov and Changhee mixed-type polynomials and give some interesting identities of those polynomials.

\section{The Korobov and Changhee mixed-type polynomials}

Let us define Korobov and Changhee mixed-type polynomials $\mathrm{KCh}_{n, q}(x)$ of the first kind as follows:

$$
K C h_{n, q}(x)=(-1)^{n} \int_{\mathbb{Z}_{p}}(q y+x)_{n} d \mu_{-1}(y), \quad(n \geqslant 0) .
$$

Then, by (1.9) and (2.1), we have

$$
\mathrm{KCh}_{n, \mathrm{q}}(\mathrm{x})=\mathrm{Ch}_{\mathrm{l}, \mathrm{q}}(\mathrm{x})(-1)^{\mathrm{n}} .
$$

By (2.1), we derive the generating function of $\mathrm{KCh}_{n, q}(\mathrm{x})$ as follows:

$$
\begin{aligned}
\sum_{n=0}^{\infty} K C h_{n, q}(x) \frac{t^{n}}{n !} & =\int_{\mathbb{Z}_{p}} \sum_{n=0}^{\infty}(q y+x)_{n} \frac{(-t)^{n}}{n !} d \mu_{-1}(y) \\
& =\int_{\mathbb{Z}_{p}} \sum_{n=0}^{\infty}\left(\begin{array}{c}
q y+x \\
n
\end{array}\right)(-t)^{n} d \mu_{-1}(y) \\
& =\int_{\mathbb{Z}_{p}}(1-t)^{q y+x} d \mu_{-1}(y) \\
& =\frac{2}{(1-t)^{q}+1}(1-t)^{x} .
\end{aligned}
$$

Note that the generating function of the Stirling number is given by

$$
\left(e^{t}-1\right)^{n}=n ! \sum_{l=n}^{\infty} S_{2}(l, n) \frac{t^{l}}{l !}, \quad(\text { see }[4,5,8,10,13,14,16,20,21]) .
$$

Recall that the Euler polynomials was defined by the generating function as follows:

$$
\int_{\mathbb{Z}_{p}} e^{(y+x) t} d \mu_{-1}(y)=\frac{2}{e^{t}+1} e^{x t}=\sum_{n=0}^{\infty} E_{n}(x) \frac{t^{n}}{n !} .
$$

Replacing $t$ by $1-e^{t}$, we have

$$
\begin{aligned}
\sum_{m=0}^{\infty} K C h_{m, q}(x)(-1)^{m} \frac{\left(e^{t}-1\right)^{m}}{m !} & =\frac{2}{1+e^{q t}} e^{\frac{x}{q} q t} \\
& =\sum_{n=0}^{\infty} E_{n}\left(\frac{x}{q}\right) q^{n} \frac{t^{n}}{n !},
\end{aligned}
$$


and

$$
\begin{aligned}
\sum_{m=0}^{\infty} K C h_{m, q}(x)(-1)^{m} \frac{\left(e^{t}-1\right)^{m}}{m !} & =\sum_{m=0}^{\infty} K C h_{m, q}(x)(-1)^{m} \sum_{n=m}^{\infty} S_{2}(n, m) \frac{t^{n}}{n !} \\
& =\sum_{n=0}^{\infty}\left(\sum_{m=0}^{n}(-1)^{m} K C h_{m, q}(x) S_{2}(n, m)\right) \frac{t^{n}}{n !} .
\end{aligned}
$$

Thus by (2.2) and (2.3), we obtain the following theorem.

Theorem 2.1. For $\mathrm{n} \geqslant 0$, we have

$$
E_{n}\left(\frac{x}{q}\right) q^{n}=\sum_{l=0}^{n}(-1)^{l} K C h_{l, q}(x) S_{2}(l, n) .
$$

In view of (2.1), we define the Korobov and Changhee mixed-type polynomials of the second kind as following:

$$
\widehat{\mathrm{KCh}}_{n, q}(x)=(-1)^{n} \int_{\mathbb{Z}_{\mathrm{p}}}(-\mathrm{q} y+x)_{n} \mathrm{~d} \mu_{-1}(y), \quad(\mathrm{n} \geqslant 0) .
$$

From (2.4), we get

$$
\begin{aligned}
\sum_{n=0}^{\infty} \widehat{\mathrm{KCh}}_{n, q} \frac{\mathrm{t}^{\mathrm{n}}}{\mathrm{n} !} & =\sum_{n=0}^{\infty}(-1)^{n} \int_{\mathbb{Z}_{p}}(-q y+x)_{n} d \mu_{-1}(y) \frac{\mathrm{t}^{\mathrm{n}}}{\mathrm{n} !} \\
& =\int_{\mathbb{Z}_{p}}(1-t)^{-q y+x} d \mu_{-1}(y) .
\end{aligned}
$$

From (1.2), we have

$$
\int_{\mathbb{Z}_{\mathrm{p}}}(1-\mathrm{t})^{-\mathrm{q} y+x} \mathrm{~d} \mu_{-1}(\mathrm{y})=\frac{2}{(1-\mathrm{t})^{-\mathrm{q}+1}}(1-\mathrm{t})^{\mathrm{x}} .
$$

By (2.5) and (2.6), we derive the generating function of $\widehat{\mathrm{KCh}}_{n, q}(x)$ as follows:

$$
\sum_{n=0}^{\infty} \widehat{\mathrm{KCh}}_{n, q}(x) \frac{\mathrm{t}^{\mathrm{n}}}{\mathrm{n} !}=\frac{2}{(1-\mathrm{t})^{-q}+1}(1-\mathrm{t})^{\mathrm{x}} .
$$

From (2.4), we have

$$
\begin{aligned}
\widehat{\mathrm{KCh}}_{n, q}(x) & =(-1)^{n} \int_{\mathbb{Z}_{p}}(-q y+x)_{n} d \mu_{-1}(y) \\
& =\sum_{l=0}^{n} S_{1}(n, l) \int_{\mathbb{Z}_{p}}(-q y+x)^{l} d \mu_{-1}(y)(-1)^{n} \\
& =\sum_{l=0}^{n} S_{1}(n, l)(-1)^{l+n} q^{l} \int_{\mathbb{Z}_{p}}\left(y-\frac{x}{q}\right)^{l} d \mu_{-1}(y) \\
& =\sum_{l=0}^{n} S_{1}(n, l)(-1)^{n+l} q^{l} E_{l}\left(-\frac{x}{q}\right) .
\end{aligned}
$$

Thus, by (2.8), we obtain the following theorem.

Theorem 2.2. For $\mathrm{n} \geqslant 0$, we have

$$
\widehat{\mathrm{KCh}}_{n, q}(x)=\sum_{l=0}^{n} S_{1}(n, l)(-1)^{n+l} q^{l} E_{l}\left(-\frac{x}{q}\right) .
$$


We observe that

$$
\begin{aligned}
\sum_{n=0}^{\infty} E_{n}(-x) \frac{t^{n}}{n !} & =\frac{2}{e^{t}+1} e^{-x t} \\
& =\frac{2}{1+e^{-t}} e^{-(x+1) t} \\
& =\sum_{n=0}^{\infty} E_{n}(1+x)(-1)^{n} \frac{t^{n}}{n !}
\end{aligned}
$$

By (2.10), we get

$$
E_{n, q}(-x)=E_{n}(1+x)(-1)^{n}
$$

By (2.8) and (2.11), we obtain the following theorem.

Theorem 2.3. For $\mathrm{n} \geqslant 0$,

$$
\widehat{\mathrm{KCh}}_{n, \mathrm{q}}(\mathrm{x})=\sum_{\mathrm{l}=0}^{\mathrm{n}} \mathrm{S}_{1}(\mathrm{n}, \mathrm{l})(-1)^{\mathrm{n}} \mathrm{q}^{\mathrm{l}} \mathrm{E}_{\mathrm{l}}\left(1+\frac{\mathrm{x}}{\mathrm{q}}\right) .
$$

By replacing $t$ by $1-e^{t}$ in (2.7)

$$
\begin{aligned}
\sum_{n=0}^{\infty} \widehat{\mathrm{KCh}}_{n, q}(x) \frac{\left(1-e^{\mathrm{t}}\right)}{n !} & =\frac{2}{1+e^{q \mathrm{t}}} e^{q \mathrm{t}} e^{\mathrm{tx}} \\
& =\frac{2}{1+e^{q \mathrm{q}}} e^{(\mathrm{q}+\mathrm{x}) \mathrm{t}} \\
& =\frac{2}{1+e^{q \mathrm{t}}} e^{\left(1+\frac{x}{q}\right) \mathrm{qt}} \\
& =\sum_{n=0}^{\infty} E_{n}\left(1+\frac{x}{q}\right) q^{n} \frac{\mathrm{t}^{\mathrm{n}}}{n !}
\end{aligned}
$$

and

$$
\begin{aligned}
\sum_{m=0}^{\infty} \widehat{K C h}_{m, q}(x) \frac{1}{m !}\left(1-e^{t}\right)^{m} & =\sum_{m=0}^{\infty} \widehat{K C h}_{m, q}(x) \frac{1}{m !} m !(-1)^{m} \sum_{n=m}^{\infty} S_{2}(n, m) \frac{t^{n}}{n !} \\
& =\sum_{n=0}^{\infty}\left(\sum_{m=0}^{n} \widehat{K C h}_{m, q}(x)(-1)^{m} S_{2}(n, m)\right) \frac{t^{n}}{n !}
\end{aligned}
$$

Thus, by (2.12) and (2.13), we obtain the following theorem.

Theorem 2.4. For $\mathrm{n} \geqslant 0$, we have

$$
E_{n}\left(1+\frac{x}{q}\right) q^{n}=\sum_{m=0}^{n} \widehat{K C h}_{m, q}(x)(-1)^{m} S_{2}(n, m) .
$$

We observe that

$$
\begin{aligned}
\frac{\widehat{\mathrm{KCh}}_{n, q}}{n !} & =\frac{(-1)^{n}}{n !} \int_{\mathbb{Z}_{\mathrm{p}}}(-q y)_{n} \mathrm{~d} \mu_{-1}(\mathrm{y}) \\
& =\int_{\mathbb{Z}_{\mathrm{p}}}\left(\begin{array}{c}
\mathrm{q} y+n-1 \\
n
\end{array}\right) \mathrm{d} \mu_{-1}(\mathrm{y}) \\
& =\sum_{l=0}^{n}\left(\begin{array}{c}
n-1 \\
n-l
\end{array}\right) \int_{\mathbb{Z}_{p}}\left(\begin{array}{c}
q y \\
l
\end{array}\right) d \mu_{-1}(y) \\
& =\sum_{l=0}^{n}\left(\begin{array}{c}
n-1 \\
n-l
\end{array}\right) \frac{1}{l !} \int_{\mathbb{Z}_{p}}(q y)_{l} d \mu_{-1}(y) \\
& =\sum_{l=0}^{n}\left(\begin{array}{c}
n-1 \\
l-1
\end{array}\right) \frac{K C h_{l, q}}{l !} .
\end{aligned}
$$


Thus, by (2.14), we obtain the following theorem.

Theorem 2.5. For $n \geqslant 1$, we have

$$
\frac{\widehat{\mathrm{KCh}}_{n, \mathrm{q}}}{\mathrm{n} !}=\sum_{l=0}^{\mathrm{n}}\left(\begin{array}{c}
\mathrm{n}-1 \\
l-1
\end{array}\right) \frac{\mathrm{KCh}}{\mathrm{l}, \mathrm{q}}
$$

\section{The Korobov and Changhee mixed-type polynomials of order $r$}

For $r \in \mathbb{N}$, let us consider Korobov and Changhee mixed-type polynomials of order $r$ as follows:

$$
K C h_{n, q}^{(r)}(x)=(-1)^{n} \int_{\mathbb{Z}_{p}} \cdots \int_{\mathbb{Z}_{p}}\left(q x_{1}+\cdots+q x_{r}+x\right)_{n} d \mu_{-1}\left(x_{1}\right) \cdots d \mu_{-1}\left(x_{r}\right) .
$$

Then we have

$$
\begin{aligned}
K C h_{n, q}^{(r)}(x) & =(-1)^{n} \int_{\mathbb{Z}_{p}} \cdots \int_{\mathbb{Z}_{p}}\left(q x_{1}+\cdots+q x_{r}+x\right)_{n} d \mu_{-1}\left(x_{1}\right) \cdots d \mu_{-1}\left(x_{r}\right) \\
& =\sum_{l=0}^{n} S_{1}(n, l)(-1)^{n} \int_{\mathbb{Z}_{p}} \cdots \int_{\mathbb{Z}_{p}}\left(q x_{1}+\cdots+q x_{r}+x\right)^{r} d \mu_{-1}\left(x_{1}\right) \cdots d \mu_{-1}\left(x_{r}\right) \\
& =\sum_{l=0}^{n} S_{1}(n, l) q^{r}(-1)^{n} \int_{\mathbb{Z}_{p}} \cdots \int_{\mathbb{Z}_{p}}\left(x_{1}+\cdots+x_{r}+\frac{x}{q}\right)^{r} d \mu_{-1}\left(x_{1}\right) \cdots d \mu_{-1}\left(x_{r}\right) .
\end{aligned}
$$

Recall that the Euler polynomials of order $r$ was defined by the generating function as follows:

$$
\int_{\mathbb{Z}_{p}} \cdots \int_{\mathbb{Z}_{\mathfrak{p}}} e^{\left(x_{1}+\cdots+x_{r}+x\right) t} d \mu_{-1}\left(x_{1}\right) \cdots d \mu_{-1}\left(x_{r}\right)=\left(\frac{2}{e^{t}+1}\right)^{r} e^{x t}=\sum_{n=0}^{\infty} E_{n}^{(r)}(x) \frac{t^{n}}{n !} .
$$

From (3.2) and (3.3), we obtain the following theorem.

Theorem 3.1. For $n \geqslant 0$, we have

$$
K C h_{n, q}^{(r)}(x)=\sum_{l=0}^{n} S_{1}(n, l) q^{r}(-1)^{n} E_{n}^{(r)}\left(\frac{x}{q}\right)
$$

From (3.1), we can derive the generating function of $\mathrm{KCh}_{n, q}(x)$ as follows:

$$
\begin{aligned}
\sum_{n=0}^{\infty} K C h_{n, q}^{(r)}(x) \frac{t^{n}}{n !} & =\sum_{n=0}^{\infty}(-1)^{n} \int_{\mathbb{Z}_{p}} \cdots \int_{\mathbb{Z}_{p}}\left(q x_{1}+\cdots+q x_{r}+x\right)_{n} d \mu_{-1}\left(x_{1}\right) \cdots d \mu_{-1}\left(x_{r}\right) \frac{t^{n}}{n !} \\
& =\int_{\mathbb{Z}_{p}} \cdots \int_{\mathbb{Z}_{p}} \sum_{n=0}^{\infty}\left(\begin{array}{c}
q x_{1}+\cdots+q x_{r}+x \\
n
\end{array}\right)(-t)^{n} d \mu_{-1}\left(x_{1}\right) \cdots d \mu_{-1}\left(x_{r}\right) \\
& =\int_{\mathbb{Z}_{p}} \cdots \int_{\mathbb{Z}_{p}}(1-t)^{q x_{1}+\cdots+q x_{r}+x} d \mu_{-1}\left(x_{1}\right) \cdots d \mu_{-1}\left(x_{r}\right) \\
& =\left(\frac{2}{(1-t)^{q}+1}\right)^{r}(1-t)^{x} .
\end{aligned}
$$

As is known, the $\mathrm{q}-$ Changhee polynomials of order $r$ are defined by the generating function to be

$$
\left(\frac{2}{(1+t)^{q}+1}\right)^{r}(1+t)^{x}=\sum_{n=0}^{\infty} C h_{n, q}^{(r)}(x) \frac{t^{n}}{n !} .
$$


By replacing $t$ by $1-e^{t}$ in (3.4)

$$
\begin{aligned}
\sum_{n=0}^{\infty} K C h_{n, q}^{(r)}(x) \frac{\left(1-e^{t}\right)^{n}}{n !} & =\sum_{n=0}^{\infty} K C h_{n, q}^{(r)}(x) \frac{1}{n !} n ! \sum_{l=n}^{\infty} S_{2}(l, n)(-1)^{n} \frac{t^{l}}{l !} \\
& =\sum_{l=0}^{\infty}\left(\sum_{n=0}^{l} K C h_{n, q}^{(r)}(x)(-1)^{n} S_{2}(l, n)\right) \frac{t^{l}}{l !}
\end{aligned}
$$

and

$$
\begin{aligned}
\sum_{n=0}^{\infty} K C h_{n, q}^{(r)}(x) \frac{\left(1-e^{t}\right)^{n}}{n !} & =\left(\frac{2}{e^{q t}+1}\right)^{r} e^{t x} \\
& =\left(\frac{2}{e^{q t}+1}\right)^{r} e^{\frac{x}{q} q t} \\
& =\sum_{l=0}^{\infty} E_{l}^{(r)} q^{l}\left(\frac{x}{q}\right) \frac{t^{l}}{l !}
\end{aligned}
$$

From (3.5) and (3.6), we obtain the following theorem.

Theorem 3.2. For $n \geqslant 0$,

$$
q^{n} E_{n}^{(r)}(x)=\sum_{l=0}^{n} K C h_{l, q}^{(r)}(x)(-1)^{l} S_{2}(n, l) .
$$

Let us consider Korobov and Changhee mixed-type polynomials of second kind with order $r$ as follows:

$$
\widehat{\mathrm{KCh}}_{n, q}^{(r)}(x)=(-1)^{n} \int_{\mathbb{Z}_{p}} \cdots \int_{\mathbb{Z}_{p}}\left(-q x_{1}-q x_{2}-\cdots-q x_{r}+x\right)_{n} d \mu_{-1}\left(x_{1}\right) \cdots d \mu_{-1}\left(x_{r}\right),
$$

where $n \geqslant 0$.

Thus, by (3.7), we get

$$
\begin{aligned}
& \widehat{\mathrm{KCh}}_{n, \mathrm{q}}^{(\mathrm{r})}(\mathrm{x})=\sum_{\mathrm{l}=0}^{\mathrm{n}} \mathrm{S}_{1}(\mathrm{n}, \mathrm{l})(-1)^{\mathrm{n}} \int_{\mathbb{Z}_{\mathrm{p}}} \cdots \int_{\mathbb{Z}_{\mathrm{p}}}\left(-\mathrm{q} \mathrm{x}_{1}-\mathrm{q} \mathrm{x}_{2}-\cdots-\mathrm{q} \mathrm{x}_{\mathrm{r}}+\mathrm{x}\right)^{\mathrm{l}} \mathrm{d} \mu_{-1}\left(\mathrm{x}_{1}\right) \cdots \mathrm{d} \mu_{-1}\left(\mathrm{x}_{\mathrm{r}}\right) \\
& =\sum_{l=0}^{n} S_{1}(n, l)(-1)^{n+l} q^{l} \int_{\mathbb{Z}_{p}} \cdots \int_{\mathbb{Z}_{p}}\left(x_{1}+\cdots+x_{r}-\frac{x}{q}\right)^{l} d \mu_{-1}\left(x_{1}\right) \cdots d \mu_{-1}\left(x_{r}\right) \\
& =\sum_{l=0}^{n} S_{1}(n, l)(-1)^{n+l} q^{l} E_{l}^{(r)}\left(-\frac{x}{q}\right)
\end{aligned}
$$

By (3.8), we obtain the following theorem.

Theorem 3.3. For $n \geqslant 0$,

$$
\widehat{\mathrm{KCh}}_{n, q}^{(r)}(x)=\sum_{l=0}^{n} S_{1}(n, l) q^{l}(-1)^{n+l} E_{l}^{(r)}\left(-\frac{x}{q}\right) .
$$

We observe that

$$
\begin{aligned}
\sum_{n=0}^{\infty} E_{n}^{(r)}(-x) \frac{t^{n}}{n !} & =\left(\frac{2}{e^{t}+1}\right)^{r} e^{-x t} \\
& =\left(\frac{2}{1+e^{-t}}\right)^{r} e^{-r t} e^{-x t} \\
& =\left(\frac{2}{1+e^{-t}}\right)^{r} e^{-(x+r) t} \\
& =\sum_{n=0}^{\infty} E_{n}^{(r)}(x+r)(-1)^{n} \frac{t^{n}}{n !} .
\end{aligned}
$$


By (3.10), we have

$$
E_{n}^{(r)}(-x)=E_{n}^{(r)}(x+r)(-1)^{n} .
$$

By (3.9) and (3.11), we obtain the following theorem.

Theorem 3.4. For $\mathrm{n} \geqslant 0$,

$$
\widehat{\mathrm{KCh}}_{n, q}^{(r)}(x)=\sum_{l=0}^{n} S_{1}(n, l) q^{l}(-1)^{n} E_{l}^{(r)}\left(\frac{x}{q}+r\right) .
$$

\section{References}

[1] A. Bayad, T. Kim, Identities for Apostol-type Frobenius-Euler polynomials resulting from the study of a nonlinear operator, Russ. J. Math. Phys., 23 (2016), 164-171. 1.1

[2] L. Carlitz, Degenerate Stirling, Bernoulli and Eulerian numbers, Utilitas Math., 15 (1979), 51-88.

[3] D. V. Dolgii, D. S. Kim, T. Kim, On Korobov polynomials of the first kind, (Russian) Mat. Sb., 208 (2017), 65-79.

[4] D. V. Dolgy, D. S. Kim, T. Kim, S.-H. Rim, Some identities of special q-polynomials, J. Inequal. Appl., 2014 (2014), 10 pages. 2

[5] D. V. Dolgy, T. Kim, H.-I. Kwon, Identities of symmetry for the higher-order Carlitz's degenerate q-Euler polynomials under the symmetry group of degree 3, Adv. Stud. Contemp. Math. (Kyungshang), 26 (2016), 595-600. 2

[6] T. Kim, q-Volkenborn integration, Russ. J. Math. Phys., 9 (2002), 288-299.

[7] T. Kim, Identities involving Laguerre polynomials derived from umbral calculus, Russ. J. Math. Phys., 21 (2014), 36-45.

[8] T. Kim, D. V. Dolgy, D. S. Kim, S.-H. Rim, A note on the identities of special polynomials, Ars Combin., 113A (2014), 97-106. 2

[9] T. Kim, D. V. Dolgy, D. S. Kim, J.-J. Seo, Differential equations for Changhee polynomials and their applications, J. Nonlinear Sci. Appl., 9 (2016), 2857-2864.

[10] D. S. Kim, T. Kim, Identities arising from higher-order Daehee polynomial bases, Open Math., 13 (2015), 196-208. 2

[11] D. S. Kim, T. Kim, Some identities of degenerate special polynomials, Open Math., 13 (2015), 380-389. 1

[12] D. S. Kim, T. Kim, Some identities of Korobov-type polynomials associated with p-adic integrals on $\mathbb{Z}_{\mathbf{p}}$, Adv. Difference Equ., 2015 (2015), 13 pages. 1

[13] T. Kim, D. S. Kim, A note on nonlinear Changhee differential equations, Russ. J. Math. Phys., 23 (2016), 88-92. 2

[14] D. S. Kim, T. Kim, D. V. Dolgy, Some properties of special polynomials, Appl. Math. Sci., 8 (2014), 8559-8564. 1, 2

[15] D. S. Kim, T. Kim, H.-I. Kwon, T. Mansour, Nonlinear differential equation for Korobov numbers, Adv. Stud. Contemp. Math. (Kyungshang), 26 (2016), 733-740.

[16] D. S. Kim, T. Kim, H.-I. Kwon, J.-J. Seo, Identities of some special mixed-type polynomials, Adv. Stud. Theor. Phys., 8 (2014), 745-754. 2

[17] D. S. Kim, T. Kim, S.-H. Lee, D. V. Dolgy, Some special polynomials and Sheffer sequences, J. Comput. Anal. Appl., 16 (2014), 702-712.

[18] D. S. Kim, T. Kim, S.-H. Rim, Some identities arising from Sheffer sequences of special polynomials, Adv. Stud. Contemp. Math. (Kyungshang), 23 (2013), 681-693.

[19] T. Kim, D. S. Kim, J.-J. Seo, H.-I. Kwon, Differential equations associated with $\lambda$-Changhee polynomials, J. Nonlinear Sci. Appl., 9 (2016), 3098-3111.

[20] T. Kim, H.-I. Kwon, J. J. Seo, Degenerate q-Changhee polynomials, J. Nonlinear Sci. Appl., 9 (2016), 2389-2393. 1.4, 2

[21] N. M. Korobov, On some properties of special polynomials, (Russian) Proceedings of the IV International Conference "Modern Problems of Number Theory and its Applications" (Russian), Tula, (2001), Chebyshevskiǔsb., 1 (2001), 40-49. 1.1, 2 